\title{
The role of c-MET inhibitors in advanced hepatocellular carcinoma: now and future
}

\author{
Hyun Young Woo, Jeong Heo \\ Department of Internal Medicine, College of Medicine, Pusan National University and Medical Research Institute, Pusan National University \\ Hospital, Busan, Republic of Korea \\ Correspondence to: Jeong Heo. Department of Internal Medicine, College of Medicine and Medical Research Institute, Pusan National University, \\ Pusan National University Hospital, 179 Gudeok-ro, Seo-Gu, Busan 49241, Republic of Korea. Email: jheo@pusan.ac.kr. \\ Comment on: Rimassa L, Assenat E, Peck-Radosavljevic M, et al. Tivantinib for second-line treatment of MET-high, advanced hepatocellular \\ carcinoma (METIV-HCC): a final analysis of a phase 3, randomised, placebo-controlled study. Lancet Oncol 2018;19:682-93.
}

Submitted Apr 17, 2020. Accepted for publication Jun 01, 2020.

doi: 10.21037/atm-20-3387

View this article at: http://dx.doi.org/10.21037/atm-20-3387

Hepatocarcinogenesis is a complex biological process associated with several genetic and epigenetic alterations (1). Multiple molecular signaling pathways are critically involved in HCC carcinogenesis, such as Ras mitogenactivated protein kinase (Ras/Raf/MAPK), receptor tyrosine, phosphatidylinositol 3-kinase (PI3K)/AKT/mammalian target of rapamycin (mTOR), Wnt/ $\beta$-catenin, Janus kinase-signal transducer activator of transcription factor (JAK/STAT), Hedgehog (HH) and Hippo (2). At present, systemic treatment approved for patients with advanced HCC are multitargeted drugs, including sorafenib, lenvatinib, regorafenib and cabozantinib, the immune checkpoint inhibitors (ICI) nivolumab and pembrolizumab, and the monoclonal VEGFR2 antibody ramucirumab (3-9).

The receptor tyrosine kinase MET and its cognate ligand hepatocyte growth factor (HGF) play an important role in tumor pathobiology, including tumor growth, survival, neoangiogenesis, invasion, and dissemination $(10,11)$. MET exon 14 (METex14) alterations occur in up to 4\% non-small cell lung cancer (NSCLC) cases. FDA approval is expected for the novel oral selective MET inhibitors capmatinib and tepotinib, which are well tolerated with rapid and sustained effects on METex14-positive NSCLC (12). However, to date, no selective single targeted drugs have been effective for HCC. Cabozantinib, a non-selective MET inhibitor that also targets VEGFR2, AXL, and RET, has been shown to improve median overall survival about 2.2-month compared with placebo in patients with advanced HCC who had received prior therapy with sorafenib (5).

In a study of the use of tivantinib, a putative MET inhibitor, for second-line treatment of MET-high, advanced hepatocellular carcinoma (METIV-HCC) (13), overall survival was not improved with tivantinib compared to placebo [8.4 vs. 9.1 months, tivantinib vs. placebo; hazard ratio (HR) 0.97; $\mathrm{P}=0.81]$. This randomized, double-blind, placebo-controlled, phase III study evaluated the efficacy and safety of oral tivantinib (120 mg twice daily) compared with placebo in patients with advanced MET-high HCC (staining intensity score of $\geq 2$ in $\geq 50 \%$ tumor cells) who had failed with previous sorafenib therapy (Table 1). The METIV-HCC study is the first phase III trial using a biomarker during screening, such that only patients with MET-high HCC were enrolled. Although this could be looked upon as a good example of patient-oriented individualized therapy, the results did not support MET inhibition as an effective treatment for patients with HCC. Biopsy specimens were required to confirm the biomarker during screening, resulting in delayed treatment and a high rate of screening failure at the time of randomization. As a result, patients with rapid disease progression may have dropped out during the enrollment period while patients with less aggressive disease were included. This may explain the longer overall survival in the placebo group compared to the phase II study group. To date, the MET inhibitory role of tivantinib has yet to be validated because MET inhibition with tivantinib was not evaluated during the METIV-HCC study. Tivantinib is a putative MET inhibitor that may suppress the viability of cancer cells through microtubule inhibition, irrespective of MET activation (15-17), a finding supported by the observation of neutropenia of 
Table 1 Trials of c-MET inhibitors for advanced HCC

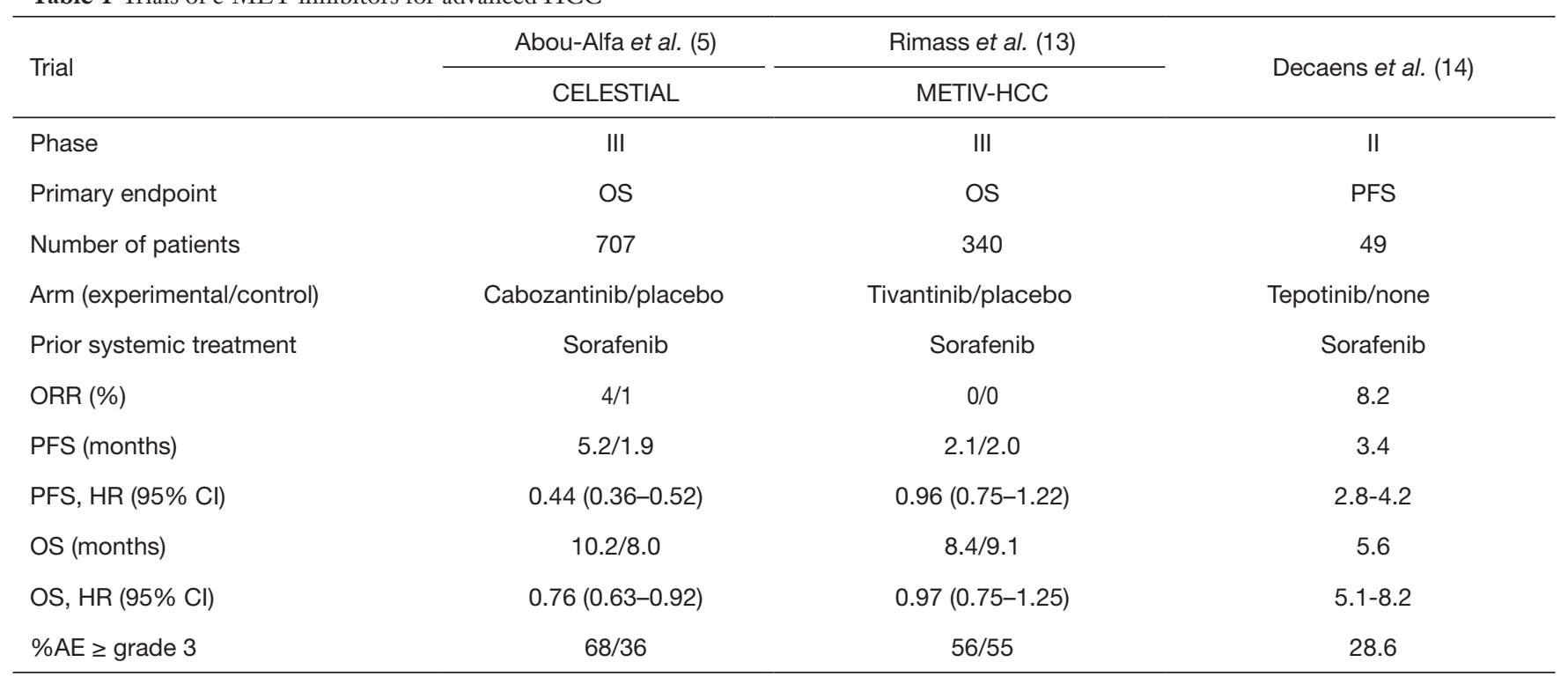

HCC, hepatocellular carcinoma; PFS, progression-free survival; OS, overall survival; HR, hazard ratio; AE, adverse events; ORR, objective response rate.

grade 3 or higher associated with tivantinib exposure in clinical trials (18). Therefore, the failure of tivantinib to improve overall survival in the METIV-HCC trial does not necessarily signify that MET inhibition was not effective as targeted therapy for HCC. Selective MET inhibitors, such as capmatinib and tepotinib, may be effective for HCC, similar to NSCLC, although further research is required to establish their efficacy. In a phase II trial with tepotinib, a selective MET inhibitor in patients with advanced Methigh HCC and previous sorafenib treatment, 31/49 (63.3\%) cases were progression-free at 12 weeks. Although the phase II data with c-MET inhibitors are encouraging, phase III studies are not expected due to their relatively modest effects and limited patient population pool (14). Another potential explanation for the negative result obtained in this phase III trial is that MET expression might not be the only factor determining resistance to sorafenib, and that inhibition of another pathway, such as the VEGF pathway, might be necessary to exert the full oncogenic effect. As mentioned above, positive results were obtained in a phase III trial of cabozantinib, a multitargeted inhibitor, which may support this hypothesis.

In general, targeting the VEGF signaling pathway with small-molecule tyrosine kinase inhibitors (TKIs) has improved the clinical outcomes of patients with advanced HCC. However, improvements have been modest, with median OS between 10.7 and 13.6 months $(4,9)$. More recently, ICI therapies have been evaluated as a potential new strategy for HCC. While single-agent ICIs have not met the requisite endpoints in phase III studies (7), promising results are reported with drug combinations. In a phase $\mathrm{Ib}$ study of atezolizumab $[1,200 \mathrm{mg}$ once every 3 weeks $(\mathrm{q} 3 \mathrm{w})]$ combined with the anti-VEGF targeting antibody, bevacizumab, among 68 efficacy-evaluable subjects with a median survival follow-up of over 18 weeks, objective response rates were confirmed in 68 patients (34\%) regardless of HCC etiology, geographic region, baseline alpha-fetoprotein (AFP) level or presence of metastasis. The median PFS was 14.9 months (95\% CI, 8.1-not estimable). The median estimates for duration of response (DOR), time to progression (TTP) and OS were not yet attained at data cutoff in Jul 2018. Treatment-related Grade 3 or 4 adverse events (AEs) were recorded in 17 subjects (25\%), most commonly hypertension $[\mathrm{n}=8(12 \%)]$. No Grade 5 AEs were observed. The high response rates indicate that the atezolizumab-bevacizumab combination exerts synergistic activity compared to early single-agent therapy with atezolizumab or bevacizumab alone in treatment-naive advanced HCC (19). Based on these encouraging results, a phase III study comparing the efficacy of atezolizumab in combination with bevacizumab vs. sorafenib in unresectable HCC patients that have received no prior systemic therapy 
is ongoing. Primary analysis disclosed an HR for overall survival of $0.58(95 \% \mathrm{CI}, 0.42-0.79 ; \mathrm{P}<0.001)$ and an HR for PFS of 0.59 (95\% CI, 0.47-0.76; $\mathrm{P}<0.001)$ for atezolizumab combined with bevacizumab $v$ s. sorafenib with a median follow-up of 8.6 months. No new safety signals were identified. The observed improvements in OS and PFS support the utility of the combination therapy as an effective novel strategy for HCC (20).

Targets of cabozantinib are also implicated in promoting tumor immune suppression, including members of the TAM family of receptor tyrosine kinases TYRO3, MER, and AXL. Preclinical (21) and clinical studies on circulating immune suppressive cells and immune effector cells in cancer patients (22) suggest that cabozantinib promotes an immune-permissive environment that may present an opportunity for synergistic effects with ICIs. For instance, in a phase Ib study (NCT03170960) currently evaluating a combination of cabozantinib [ 40 and $60 \mathrm{mg}$ orally once daily (qd)] and atezolizumab (1,200 $\mathrm{mg}$ IV q3w) in multiple tumor cohorts, confirmed ORR was $70 \%$ with 1 complete response (CR) and 6 PRs, along with no Grade 4 or 5 AEs. Based on the favorable safety profile and preliminary efficacy data, a phase III study evaluating the safety and efficacy of cabozantinib combined with atezolizumab versus standard-of-care sorafenib in subjects with advanced HCC with no previous exposure to systemic anticancer therapy is ongoing (23).

We are yet to establish whether combination of tivantinib or tepotinib with ICI can exert a synergistic effect as in the case of anti-angiogenesis agents. In a recent report (24), the expression of PD ligand 1 (PDL1) was enhanced and co-cultured $\mathrm{T}$ cells was inactivated when MET inhibitors exposed to liver cancer cell lines. Notably, however, tumor growth was suppressed and survival was prolonged with combination of anti-PD1 compared with anti-PD1 or MET inhibitors alone. These results highlight the possibility that treatment of HCC with a combination of c-MET inhibitors and ICI could effectively induce synergistic therapeutic effects without anti-angiogenetic activity. Future research efforts should focus on optimizing the antitumor effects of c-MET inhibitors.

\section{Acknowledgments}

Funding: This research was supported by clinical research grant from Pusan National University Hospital in 2018.

\section{Footnote}

Provenance and Peer Review: This article was commissioned by the editorial office, Annals of Translational Medicine. The article did not undergo external peer review.

Conflicts of Interest: Both authors have completed the ICMJE uniform disclosure form (available at http://dx.doi. org/10.21037/atm-20-3387). The authors have no conflicts of interests to declare.

Ethical Statement: The authors are accountable for all aspects of the work in ensuring that questions related to the accuracy or integrity of any part of the work are appropriately investigated and resolved.

Open Access Statement: This is an Open Access article distributed in accordance with the Creative Commons Attribution-NonCommercial-NoDerivs 4.0 International License (CC BY-NC-ND 4.0), which permits the noncommercial replication and distribution of the article with the strict proviso that no changes or edits are made and the original work is properly cited (including links to both the formal publication through the relevant DOI and the license). See: https://creativecommons.org/licenses/by-nc-nd/4.0/.

\section{References}

1. Liu M, Jiang L, Guan XY. The genetic and epigenetic alterations in human hepatocellular carcinoma: a recent update. Protein Cell 2014;5:673-91.

2. Dimri M, Satyanarayana A. Molecular Signaling Pathways and Therapeutic Targets in Hepatocellular Carcinoma. Cancers (Basel) 2020;12:491.

3. El-Khoueiry AB, Sangro B, Yau T, et al. Nivolumab in patients with advanced hepatocellular carcinoma (CheckMate 040): an open-label, non-comparative, phase $1 / 2$ dose escalation and expansion trial. Lancet 2017;389:2492-502.

4. Kudo M, Finn RS, Qin S, et al. Lenvatinib versus sorafenib in first-line treatment of patients with unresectable hepatocellular carcinoma: a randomised phase 3 noninferiority trial. Lancet 2018;391:1163-73.

5. Abou-Alfa GK, Meyer T, Cheng AL, et al. Cabozantinib in Patients with Advanced and Progressing Hepatocellular Carcinoma. N Engl J Med 2018;379:54-63. 
6. Zhu AX, Kang YK, Yen CJ, et al. Ramucirumab after sorafenib in patients with advanced hepatocellular carcinoma and increased alpha-fetoprotein concentrations (REACH-2): a randomised, double-blind, placebocontrolled, phase 3 trial. Lancet Oncol 2019;20:282-96.

7. Finn RS, Ryoo BY, Merle P, et al. Pembrolizumab (Pembro) therapy vs best supportive care (BSC) in advanced hepatocellular carcinoma (HCC): KEYNOTE-240. Ann Oncol 2019;30 Suppl 4:iv135-iv6.

8. Bruix J, Qin S, Merle P, et al. Regorafenib for patients with hepatocellular carcinoma who progressed on sorafenib treatment (RESORCE): a randomised, double-blind, placebo-controlled, phase 3 trial. Lancet 2017;389:56-66.

9. Llovet JM, Ricci S, Mazzaferro V, et al. Sorafenib in advanced hepatocellular carcinoma. N Engl J Med 2008;359:378-90.

10. Cecchi F, Rabe DC, Bottaro DP. Targeting the HGF/Met signaling pathway in cancer therapy. Expert Opin Ther Targets 2012;16:553-72.

11. Gherardi E, Birchmeier W, Birchmeier C, et al. Targeting MET in cancer: rationale and progress. Nat Rev Cancer 2012;12:89-103.

12. Wu YL, Soo RA, Locatelli G, et al. Does c-Met remain a rational target for therapy in patients with EGFR TKIresistant non-small cell lung cancer? Cancer Treat Rev 2017;61:70-81.

13. Rimassa L, Assenat E, Peck-Radosavljevic M, et al. Tivantinib for second-line treatment of MET-high, advanced hepatocellular carcinoma (METIV-HCC): a final analysis of a phase 3, randomised, placebo-controlled study. Lancet Oncol 2018;19:682-93.

14. Decaens T, Barone C, Assenat E, et al. Efficacy and safety of the Met inhibitor tepotinib in patients (pts) with advanced Met1 hepatocellular carcinoma (HCC) previously treated with sorafenib. Ann Oncol 2018;29:1x48.

15. Rimassa L, Abbadessa G, Personeni N, et al. Tumor and circulating biomarkers in patients with second-line hepatocellular carcinoma from the randomized phase II study with tivantinib. Oncotarget 2016;7:72622-33.

Cite this article as: Woo HY, Heo J. The role of c-MET inhibitors in advanced hepatocellular carcinoma: now and future. Ann Transl Med 2020;8(23):1617. doi: 10.21037/atm-203387
16. Watermann I, Schmitt B, Stellmacher F, et al. Improved diagnostics targeting c-MET in non-small cell lung cancer: expression, amplification and activation? Diagn Pathol 2015;10:130.

17. Katayama R, Aoyama A, Yamori T, et al. Cytotoxic activity of tivantinib (ARQ 197) is not due solely to c-MET inhibition. Cancer Res 2013;73:3087-96.

18. Bouattour M, Raymond E, Qin S, et al. Recent developments of c-Met as a therapeutic target in hepatocellular carcinoma. Hepatology 2018;67:1132-49.

19. Lee K, Hsu C, Lee MS, et al. Atezolizumab + bevacizumab in hepatocellular carcinoma (HCC): Safety and clinical activity results from a phase $\mathrm{Ib}$ study. Ann Oncol 2018;29:ix47.

20. Cheng A, Qin S, Ikeda M, et al. IMbrave150: Efficacy and safety results from a ph III study evaluating atezolizumab (atezo) + bevacizumab (bev) vs sorafenib (Sor) as first treatment (tx) for patients (pts) with unresectable hepatocellular carcinoma (HCC). Ann Oncol 2019;30:ix186-ix202.

21. Kwilas AR, Ardiani A, Donahue RN, et al. Dual effects of a targeted small-molecule inhibitor (cabozantinib) on immune-mediated killing of tumor cells and immune tumor microenvironment permissiveness when combined with a cancer vaccine. J Transl Med 2014;12:294.

22. Yavuz S, Apolo AB, Kummar S, et al. Cabozantinibinduced thyroid dysfunction: a review of two ongoing trials for metastatic bladder cancer and sarcoma. Thyroid 2014;24:1223-31.

23. Rimassa L, Cheng A, Braiteh F, et al. Phase 3 (COSMIC-312) study of cabozantinib in combination with atezolizumab vs sorafenib in patients with advanced hepatocellular carcinoma (aHCC) who have not received previous systemic anticancer therapy. Ann Oncol 2019;30 Suppl 4:iv65-iv66.

24. Li H, Li CW, Li X, et al. MET inhibitors promote liver tumor evasion of the immune response by stabilizing PDL1. Gastroenterology 2019;156:1849-61.e13. 